\title{
Numerical Analysis of Liquid Mixing in a T-Micromixer with Taylor Dispersion Obstructions
}

\author{
T. Manoj Dundi \\ Mechanical Engineering Department, \\ National Institute of Technology Warangal, \\ Telangana State - 506004, India. \\ S. Chandrasekhar \\ Mechanical Engineering Department, \\ National Institute of Technology Warangal, \\ Telangana State - 506004, India. \\ Shasidar Rampalli \\ Mechanical Engineering Department, \\ National Institute of Technology Warangal, \\ Telangana State - 506004, India. \\ V. R. K. Raju \\ Mechanical Engineering Department, \\ National Institute of Technology Warangal, \\ Telangana State - 506004, India. \\ Corresponding author: vrkraju9575@gmail.com

\section{V.P. Chandramohan} \\ Mechanical Engineering Department, \\ National Institute of Technology Warangal, \\ Telangana State - 506004, India.
}

(Received January 3, 2019; Accepted August 20, 2019)

\begin{abstract}
Passive micromixers are of great importance in biomedical engineering (lab-on-chips) and chemical processing (microreactors) fields. Various hydrodynamic principles such as lamination, flow separation, and chaotic advection were employed previously to improve mixing in passive mixers. However, mixing enhancement due to velocity gradients in the flow, which is known as the Taylor dispersion effect, has been seldom studied. In the present study, thin rectangular slabs oriented in the flow direction are placed in the mixing channel of a Tmicromixer. The thin rectangular slabs are referred to as Taylor Dispersion Obstructions (TDOs) as they are designed to create velocity gradients in the flow. The mixing performance of T-micromixer with and without TDOs is estimated in the $R e$ range of 0 to 350. It is observed that there is no effect on mixing in the presence of TDOs in the low $R e(0<R e<100)$, as the velocity gradients created in the flow are considerably small. The vortex formed in the flow for $R e$ of 100 to 220 damped the gradients of velocity created in the flow (due to the presence of TDOs) which resulted in negligible improvement in the quality of mixing. However, considerable enhancement in mixing performance is obtained at high $\operatorname{Re}(250$ to 350) with the presence of TDOs in the mixer. The increase in inertial effects at higher Recreated larger gradients of velocity near the walls of TDOs and mixing channel walls and thereby a significant enhancement in mixing performance is obtained due to Taylor dispersion.
\end{abstract}

Keywords- CFD, micromixer, mixing quality, obstructions, Taylor dispersion, T-mixer. 
International Journal of Mathematical, Engineering and Management Sciences

Vol. 5, No. 1, 147-160, 2020

https://doi.org/10.33889/IJMEMS.2020.5.1.013

\section{Introduction}

Micromixing is an essential function for many important applications in the field of bio-medical and bio-chemical engineering. The most important advantages of micromixers as compared to their counterparts (macro batch reactors) are low cost of manufacturing, rapid analysis, consumption of very small amount of expensive reagents and improved portability. In biomedical engineering, micromixers are used in the applications of protein folding studies (Chan et al., 1997), DNA micro-arrays (Zhang et al., 2007), and cell separation and detection (Lu et al., 2006). In chemical processing, they are utilized for organic synthesis (Suga et al., 2003), extraction (Freitas et al., 2005) and chemical production (Bayer et al., 2004). Micromixers are broadly of two types; active and passive mixers. The active type mixers utilize an external energy source like magneto hydrodynamic (Bau et al., 2001), ultrasonic (Yaralioglu et al., 2004) or electro-kinetic (Chen and Cho, 2008) instabilities to induce mixing in the microchannel. The passive type mixers do not require any external energy source except the pressure force to drive the samples into mixing microchannel. The flow passages of the passive mixer (Buchegger et al., 2011; Chung et al., 2010; Nimafar et al., 2012) are designed in such a way that stretching and folding of samples are generated to decrease the interfacial distance between them and enhance mixing. The majority of micromixers utilized in various applications belong to passive type mixers as they are robust, easy to integrate into complex microsystems and cheap compared to active type mixers.

The efficiency of passive mixers can be very poor if mixing relies solely on the mechanism of pure diffusion. Mixing of liquids is more challenging due to their low diffusion coefficients (order of $10^{-9}$ ). Therefore, many passive mixers are designed to enhance mixing using various hydrodynamic principles (Hardt et al., 2004) such as sequential lamination, flow separation, and chaotic advection. Hessel et al. (2003) constructed three different interdigital micromixers viz., slit-type, rectangular and triangular mixers and conducted experiments for different flow rates. They have optimized the mixers and observed an increase in the yield of nearly $25 \%$ with triangular interdigital micromixer. The flow separation behind triangular obstacles in a passive mixer with convergent-divergent walls (Heshmatnezhad et al., 2016) considerably enhanced mixing. The staggered herringbone (Stroock et al., 2002) and Tesla structures (Hong et al., 2004) of microchannels generated chaotic advection and significantly improved the efficiency of micromixer. Various studies have also been done to analyze mixing in passive T-micromixer. Engler et al. (2004), in their experimental and numerical investigations, observed three different regimes of liquid mixing in T-mixer viz., laminar, vortex and engulfment in the $R e$ of 0 to 200 . They found a considerable enhancement in mixing in the range of $150<R e<200$ due to the engulfment regime. Dreher et al. (2009) carried out a study in T-mixer to characterize mixing phenomenon for different $R e$ of 0.01 to 1000 . They observed a considerable drop in the quality of mixing in T-mixer at higher $\operatorname{Re}(\operatorname{Re}>500)$ due to weak chaotic and pulsating flow.

It is found that mixing efficiency of passive mixer is improved using lamination (Hessel et al., 2003), flow separation (Heshmatnezhad et al., 2016) and chaotic advection (Hong et al., 2004; Stroock et al., 2002). However, studies on the principle of Taylor dispersion for mixing enhancement seem to be very limited. Even though the mixing performance of passive T-mixer improved at higher flow rates (Engler et al., 2004), it is remarkably reduced for $R e$ above 500. In the present study, thin rectangular slabs referred to as Taylor Dispersion Obstructions (TDOs) are positioned in random but periodic manner in the outlet channel to create velocity gradients in the flow. To study the effect of Taylor dispersion, the performance of mixing in T-mixer with TDOs is estimated for different $R e$ of 0 to 350 . 
International Journal of Mathematical, Engineering and Management Sciences

Vol. 5, No. 1, 147-160, 2020

https://doi.org/10.33889/IJMEMS.2020.5.1.013

\section{Methodology}

\subsection{Mathematical Modelling}

The equations governing the flow of fluid in the micromixer (continuity and Navier-Stokes) are given by Eqs. (1) and (2) respectively,

$\nabla \cdot V=0$

$V \cdot \nabla V=-\frac{1}{\rho} \nabla P+v \nabla^{2} V$

where ' $V$ ' denotes velocity vector, ' $\rho$ ' denotes density, ' $p$ ' denotes pressure and ' $v$ ' denotes kinematic viscosity of the fluid.

The governing equation for mass transfer in the micromixer is given by diffusion-convection equation (Eq. (3)).

$(V \cdot \nabla) c=D \nabla^{2} c$

where ' $c$ ' denotes concentration and ' $D$ ' denotes diffusion coefficient.

Mixing quality ' $\alpha$ ' is evaluated by using Eq. (4)

$\alpha=1-\sqrt{\frac{\sigma_{M}^{2}}{\sigma_{\max }^{2}}}$

where $\sigma_{\max }^{2}$ denotes maximum variance of concentration in mixture $(0.5)$ and $\sigma_{M}^{2}$ denotes variance of concentration in mixture at a cross section specified by Eq. (5)

$\sigma_{M}^{2}=\frac{1}{n} \sum_{i=1}^{n}\left(c_{i}-\bar{c}_{M}\right)^{2}$

where, $\bar{c}_{M}$ denotes mean value of concentration taken over $n$ elements of grid in a given cross section considered. If mixing quality ' $\alpha$ ' is 'zero', it implies no mixing and 'one' implies complete mixing.

\subsection{Numerical Modelling}

The geometry of T-mixer with TDOs, grid generation and simulation of mixing in the T-mixer with TDOs is carried out in ANSYS Fluent 15. The 3D sketch of T-mixer with TDOs with selected dimensions is shown in Figure 1.

Figure 2 shows hexahedral elements used in meshing whole domain of ' $\mathrm{T}$ ' geometry. These hexahedral elements line up with the direction of flow which leads to better accuracy. It also results in fewer elements as compared to the use of tetrahedral elements to mesh the same geometry. This reduces the computational effort. 
International Journal of Mathematical, Engineering and Management Sciences

Vol. 5, No. 1, 147-160, 2020

https://doi.org/10.33889/IJMEMS.2020.5.1.013

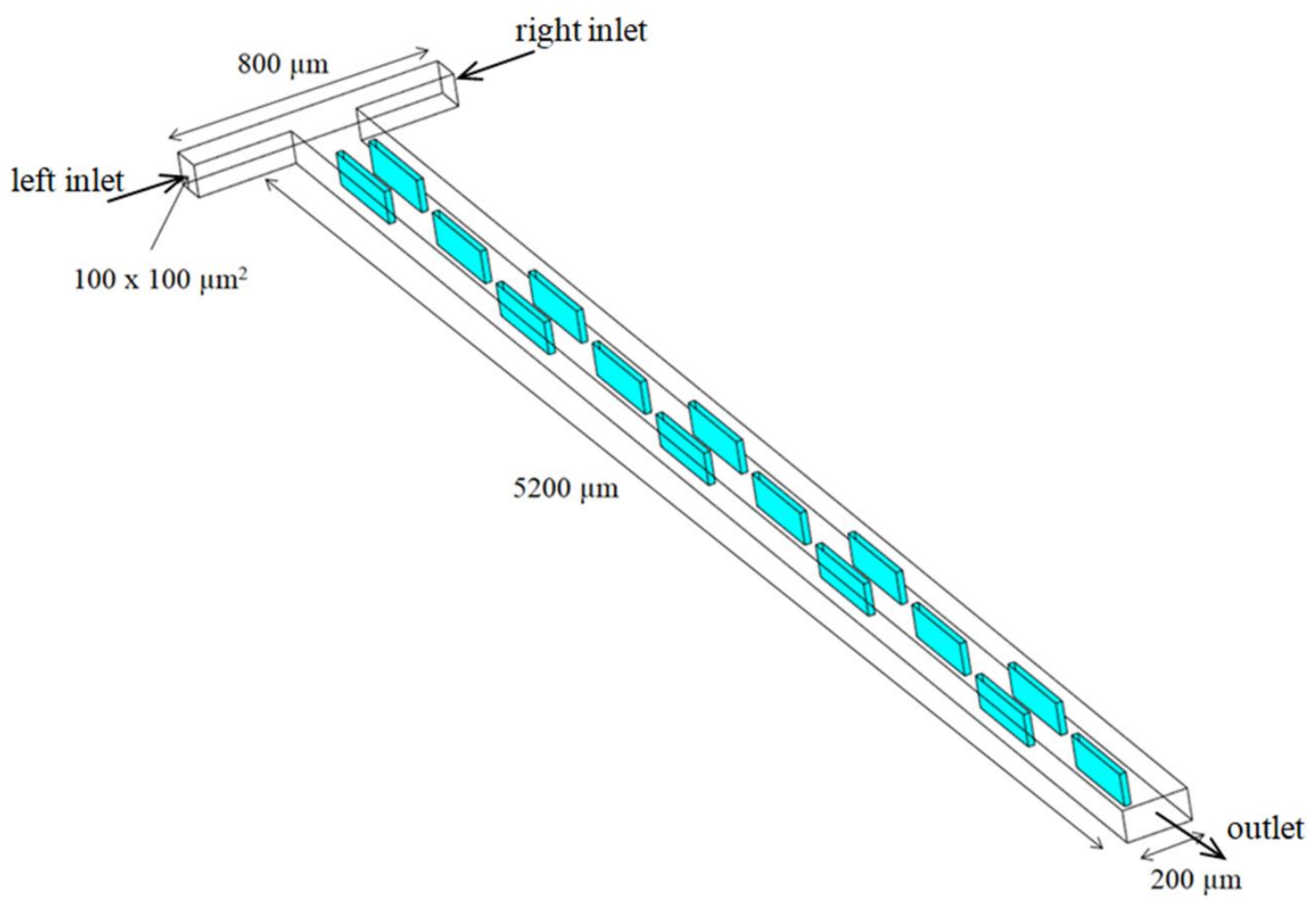

Figure 1. 3-D schematic of T-mixer with TDOs

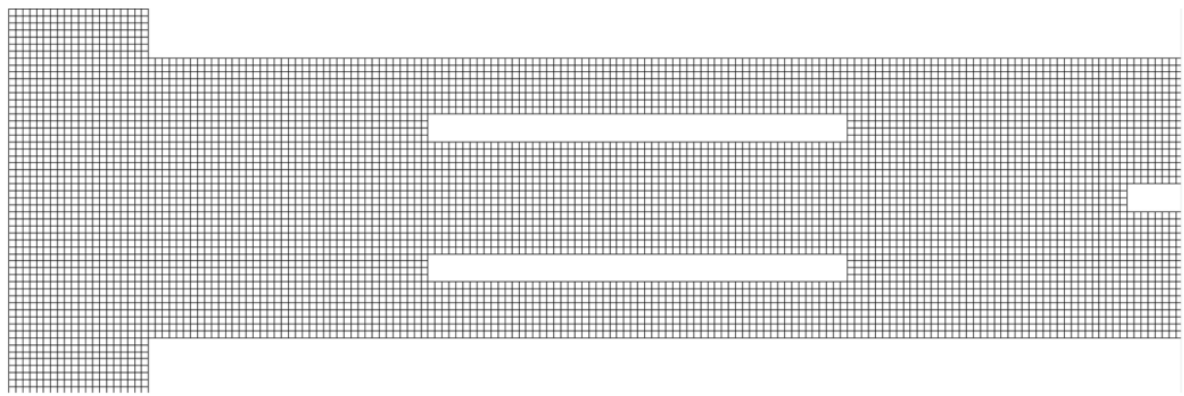

Figure 2. Grid of T-mixer with TDOs

Two liquid species (' $a$ ', ' $b$ ') are selected with properties similar to water at a temperature of $20^{\circ} \mathrm{C}$ (dynamic viscosity $\mu=0.001$ Pas, density $\rho=998.2 \mathrm{~kg} / \mathrm{m}^{3}$, and diffusion coefficient $D=2 \times 10^{-9}$ $\mathrm{m}^{2} / \mathrm{s}$ ), to be mixed in the micromixer for all the simulations. The boundary condition is given in terms of species ' $b$ ' mass fraction for the value of concentration as 'one' on left side inlet, 'zero' on right side inlet and 'zero point five' on outlet. It is set to 'zero' diffusive flux at walls. Atmospheric pressure is given as the pressure boundary condition on outlet. At inlets, the boundary condition for pressure is derived from the corresponding input velocities. The velocity 
boundary conditions are provided in Table 1. No slip velocity condition is assumed at walls of mixing channel and TDOs. Steady laminar flow and species transport models are used in solving the fluid flow and mass transfer. Table 2 shows different spatial discretization methods and schemes employed in solving the primary variables of governing equations. The convergence criterion for the residuals to monitor imbalance in the conserved equations is given as $10^{-6}$ on continuity equation, $10^{-5}$ on equations of species and momentum.

Table 1. Boundary conditions used in numerical simulations

\begin{tabular}{|c|c|c|c|}
\hline \multirow{2}{*}{ Case } & Velocity at left inlet $(\mathrm{m} / \mathrm{s})$ & Velocity at right inlet $(\mathrm{m} / \mathrm{s})$ & Reynolds number \\
& & & 2 \\
\hline I & 0.015 & 0.015 & 6 \\
\hline II & 0.045 & 0.045 & 10 \\
\hline III & 0.08 & 0.08 & 33 \\
\hline IV & 0.25 & 0.25 & 66 \\
\hline V & 0.5 & 0.5 & 106 \\
\hline VI & 0.8 & 0.8 & 133 \\
\hline VII & 1 & 1 & 146 \\
\hline VIII & 1.1 & 1.1 & 212 \\
\hline IX & 1.2 & 1.2 & 265 \\
\hline X & 1.6 & 1.6 & 318 \\
\hline XI & 2 & 2 & 345 \\
\hline XII & 2.4 & 2.4 & \\
\hline XIII & 2.6 & 2.6 & \\
\hline
\end{tabular}

Table 2. The solution methods of numerical simulation

\begin{tabular}{|l|l|}
\hline Pressure-velocity coupling & SIMPLEC \\
\hline Pressure & Standard \\
\hline Momentum & Second order Upwind scheme \\
\hline Species & Second order Upwind scheme \\
\hline
\end{tabular}

\section{3. Grid Independence Test}

As mentioned above, a commercial numerical tool, ANSYS Fluent 15 is employed to simulate the fluid flow and concentration fields in the T-mixer with TDOs. Grid independence test was conducted to arrive at the required mesh size to carry out accurate simulations of fluid flow and mass transfer in the micromixer. The variation of axial velocity at the centreline of outlet of Tmicromixer with TDOs at a $R e$ of 320 is shown in Figure 3. The maximum deviation in the axial velocity obtained for 6 and 5-micron mesh size elements is $1.3 \%$, whereas, for 5 and 4-micron mesh size elements, it is only about $0.8 \%$. Therefore, a uniform grid with 5-micron size element is selected for the domain with a total of 824000 elements. 
International Journal of Mathematical, Engineering and Management Sciences

Vol. 5, No. 1, 147-160, 2020

https://doi.org/10.33889/IJMEMS.2020.5.1.013

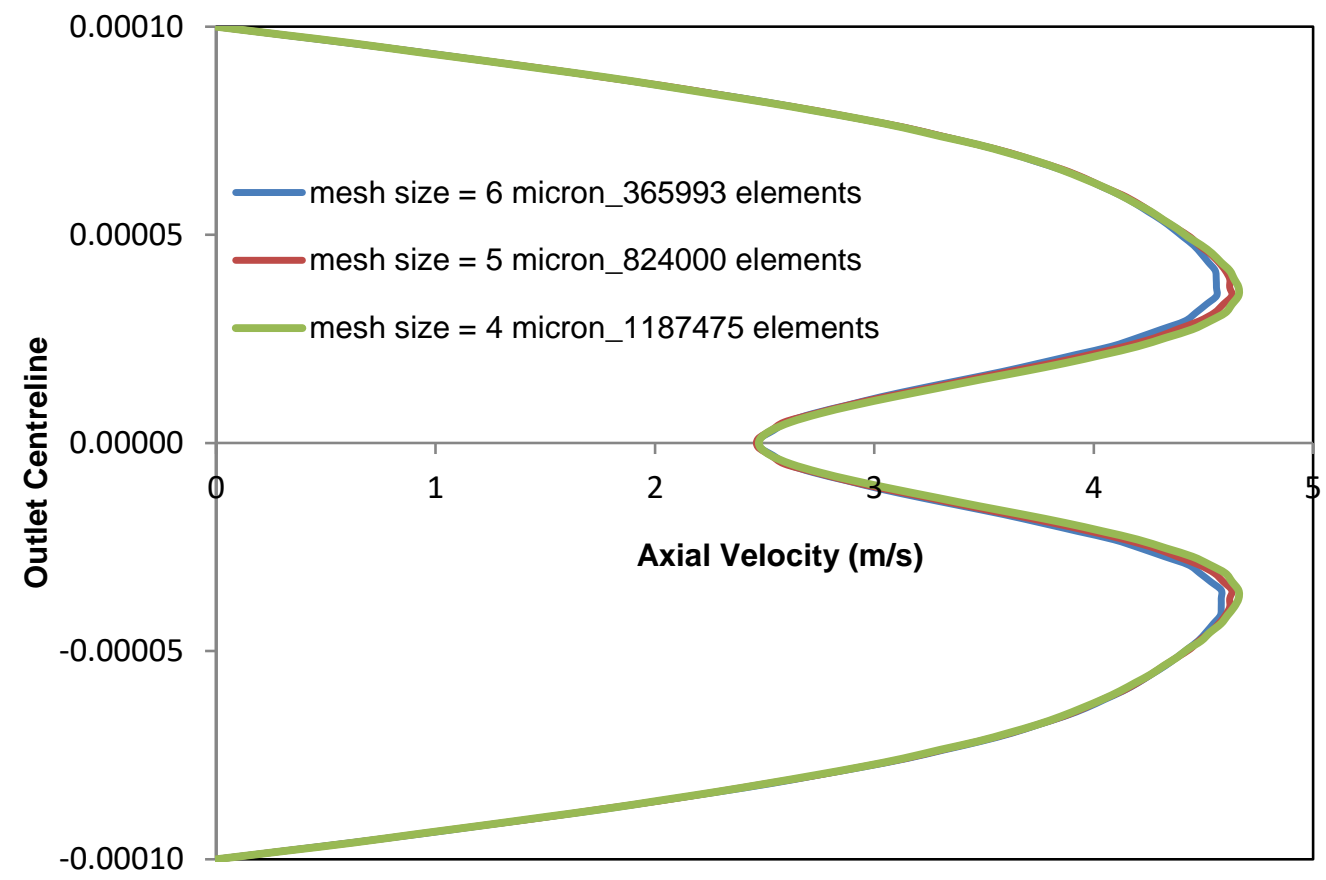

Figure 3. Axial velocity profiles on the outlet centreline of T-mixer with TDOs at $R e=318$

\section{Results and Discussion}

\subsection{Basic T-mixer}

At first, the performance of mixing in T-mixer is estimated and analysed for $R e$ of 0 to 320 . In the lower $R e$ (0 to 66), the flow in T-micromixer exhibits a completely laminar behaviour (Figure 4). Therefore, mixing takes place only due to diffusion at the little interface available in the contact of samples along the axis in the flow direction of mixing channel (Figure 5). In the Re range of 100 to 160; the vortices are formed at the confluence where the samples collide in T-mixer (Figure 6). However, the mixing has further decreased (Figure 7) as the residence time for samples has reduced. Even though vortices are formed, that is only in the self-rotation of samples confining them to the same side (left/right) of their entrance in the mixing channel (Figure 6). On further increase of $R e$, the break-up of vortices at the confluence of the channels occurred (Figure 8). The engulfment of samples taking place at the confluence has drastically improved the quality of mixing in T-mixer (Figure 9) and engulfment regime continued for Re between 180 to 320. 
International Journal of Mathematical, Engineering and Management Sciences

Vol. 5, No. 1, 147-160, 2020

https://doi.org/10.33889/IJMEMS.2020.5.1.013

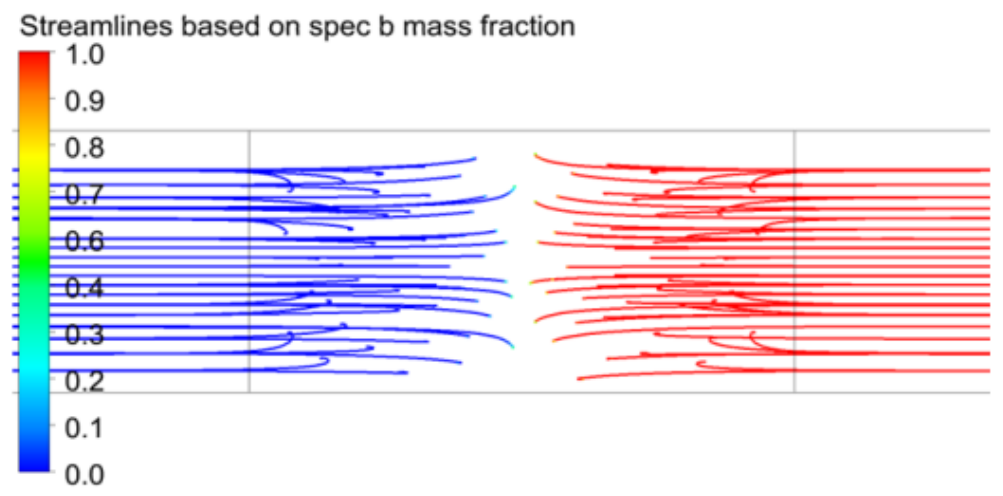

Figure 4. Streamlines at the junction of T-mixer at $R e=66$

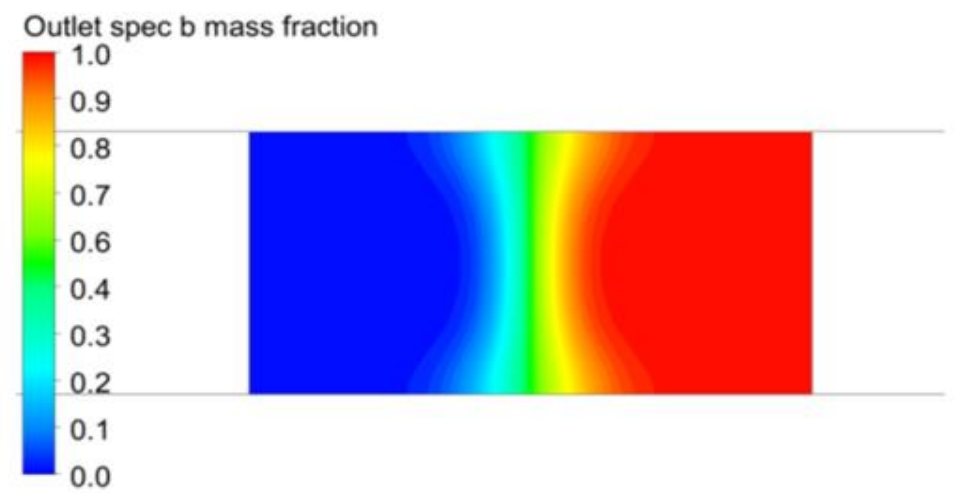

Figure 5. Mass fraction contour at outlet of T-mixer at $R e=66$

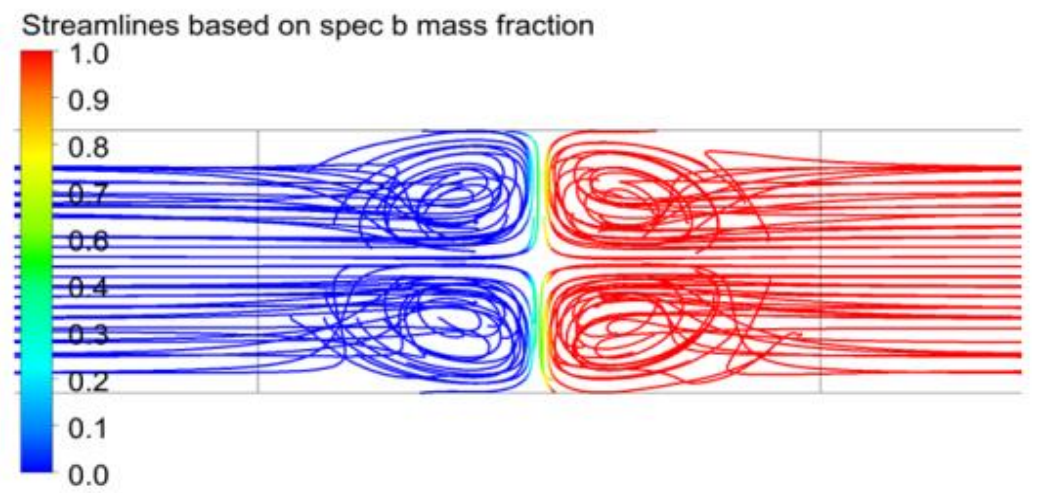

Figure 6. Streamlines at the junction of T-mixer at $R e=160$ 
International Journal of Mathematical, Engineering and Management Sciences

Vol. 5, No. 1, 147-160, 2020

https://doi.org/10.33889/IJMEMS.2020.5.1.013

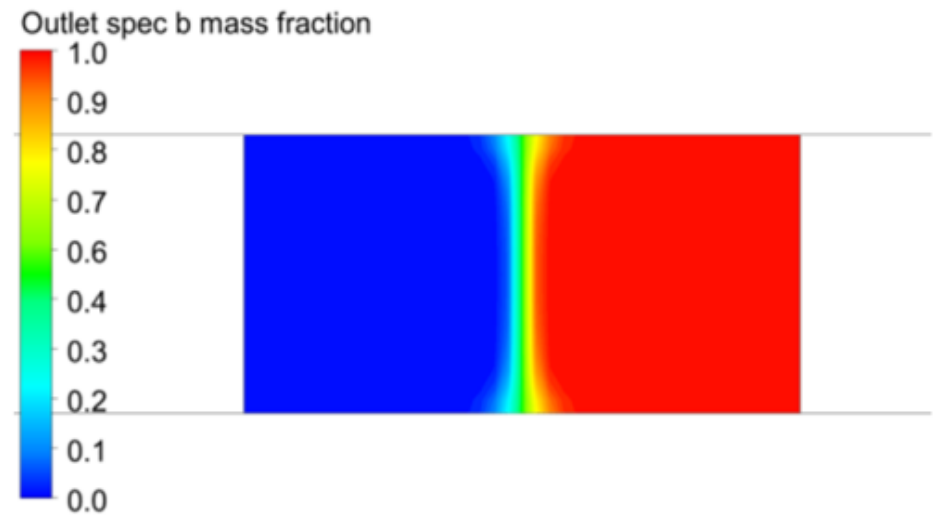

Figure 7. Mass fraction contour at outlet of T-mixer at $\mathrm{Re}=160$

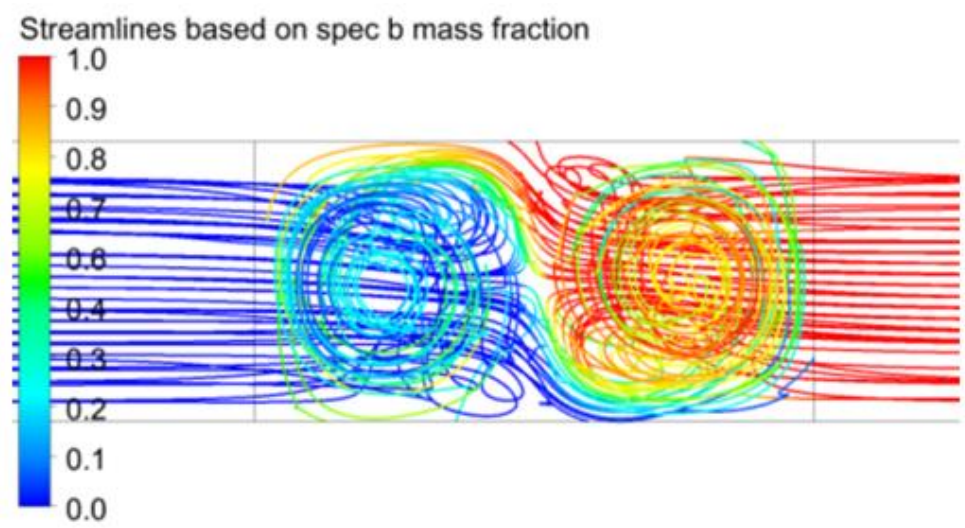

Figure 8. Streamlines at the junction of T-mixer at $R e=266$

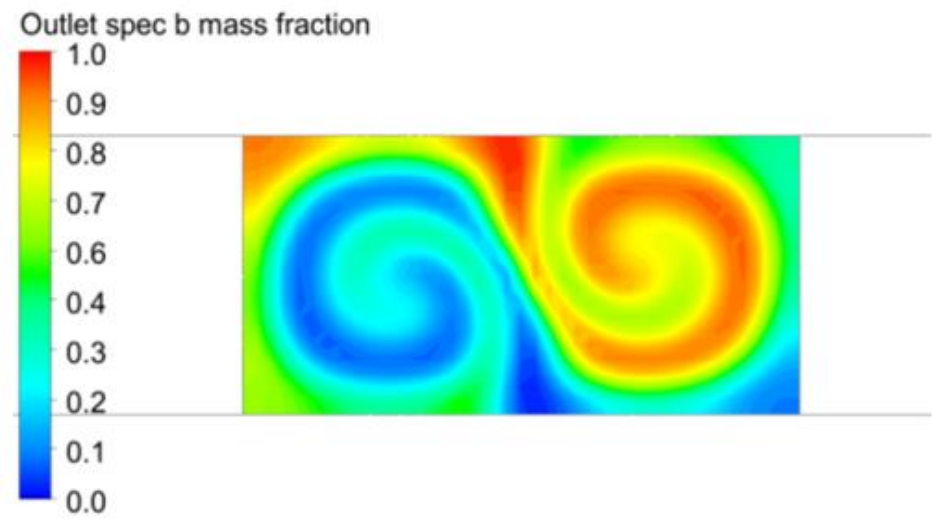

Figure 9. Mass fraction contour at outlet of T-mixer at $R e=266$ 
International Journal of Mathematical, Engineering and Management Sciences

Vol. 5, No. 1, 147-160, 2020

https://doi.org/10.33889/IJMEMS.2020.5.1.013

\subsection{T-Mixer with Taylor Dispersion Obstructions}

The performance of mixing in T-mixer with obstructions of Taylor dispersion type was evaluated in the $R e$ range of 0 to 350 . The influence of TDOs in the mixing channel at low $R e$ flows (0 to 150 ) is considerably small. However, a major influence on the mixing phenomenon has been observed particularly at high $R e$ flows of 250 to 350, with the existence of TDOs in the mixing channel.

\subsubsection{Low $\operatorname{Re}$ Flows}

In the $R e$ range of 0 to 66 , the presence of TDOs in the mixing channel produced a negligibly small effect on the performance of mixing in T-mixer (Figure 10). It is found that at lower $\operatorname{Re}(0$ to 100), the velocity gradients created in the flow due to the presence of TDO (Taylor Dispersion Obstruction) are smaller in amplitude and not significant enough to create axial dispersion and enhance the mixing. In the $R e$ range of 100 to 150 , even though there is some increase in the inertial effects, only a little increment in the quality of mixing is achieved. This is due to the fact that the vortices generated in the flow damped the velocity gradients near the surface of TDOs. Therefore, there is no increment in the quality of mixing in T-mixer with TDOs (Figure 11) compared to T-mixer without TDOs (Figure 12).

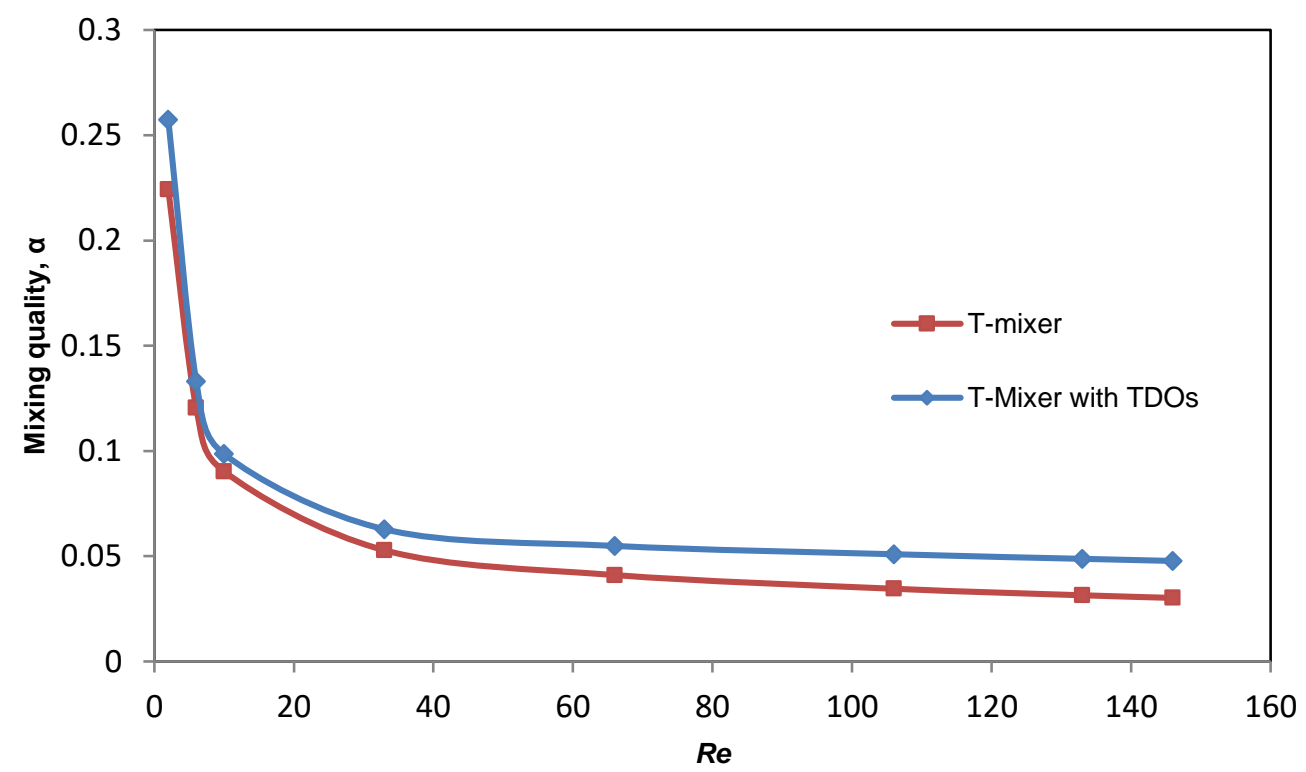

Figure 10. Variation of mixing quality with Re number 
International Journal of Mathematical, Engineering and Management Sciences

Vol. 5, No. 1, 147-160, 2020

https://doi.org/10.33889/IJMEMS.2020.5.1.013

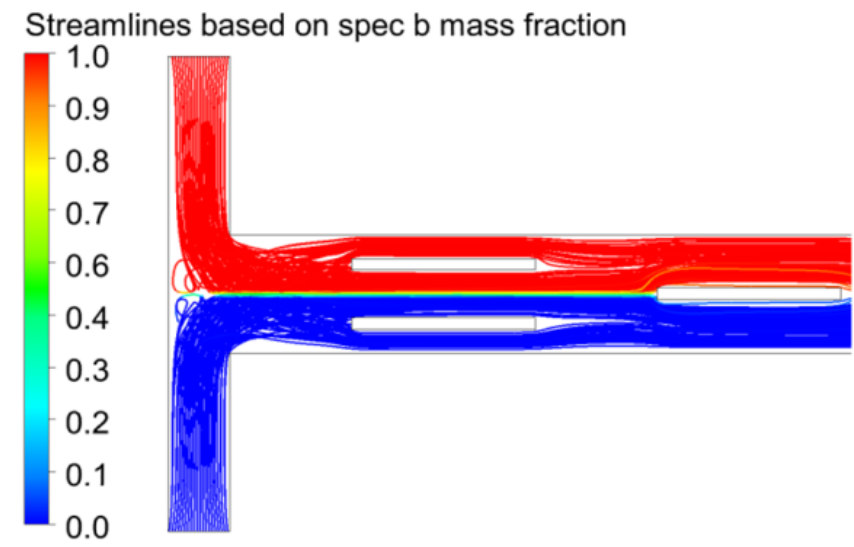

Figure 11. Streamlines of T-mixer with TDOs from top view at $R e=133$

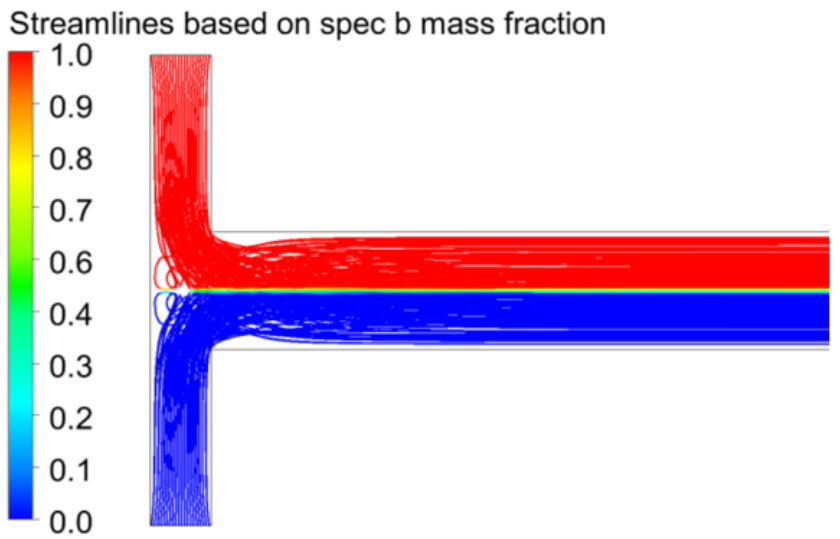

Figure 12. Streamlines of T-mixer from top view at $R e=133$

\subsubsection{High $\operatorname{Re}$ Flows}

In the $R e$ range of 150 to 220 , clearly, the engulfment flow is the most dominating effect of convective mixing in T-mixer. Therefore, the presence of TDOs in the mixing channel did not show any substantial increment in the performance of mixing in T-mixer (Figure 13). On further increase of flow rate $(R e>220)$, the quality of mixing significantly increased with the existence of TDOs in the T-mixer (Figure 14) compared to T-mixer without TDOs (Figure 15). At higher $\operatorname{Re}(\operatorname{Re}>220)$, the engulfment effects are slowly reduced in the T-mixer (Figure 16) and the inertial effects dominated. Hence, the effect of velocity gradients (Taylor dispersion) has a major influence on the flow (Figure 17) to improve mixing significantly in higher $R e$ flows (250 to $350)$. 
International Journal of Mathematical, Engineering and Management Sciences

Vol. 5, No. 1, 147-160, 2020

https://doi.org/10.33889/IJMEMS.2020.5.1.013

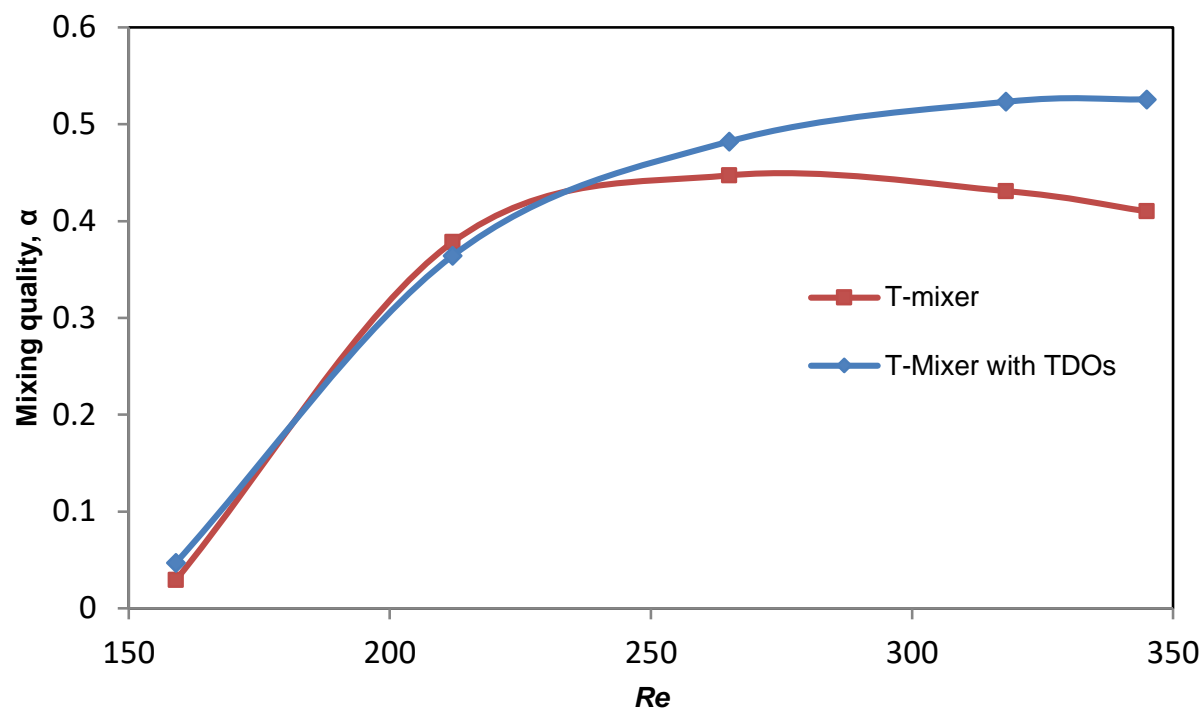

Figure 13. Variation of mixing quality with Re number

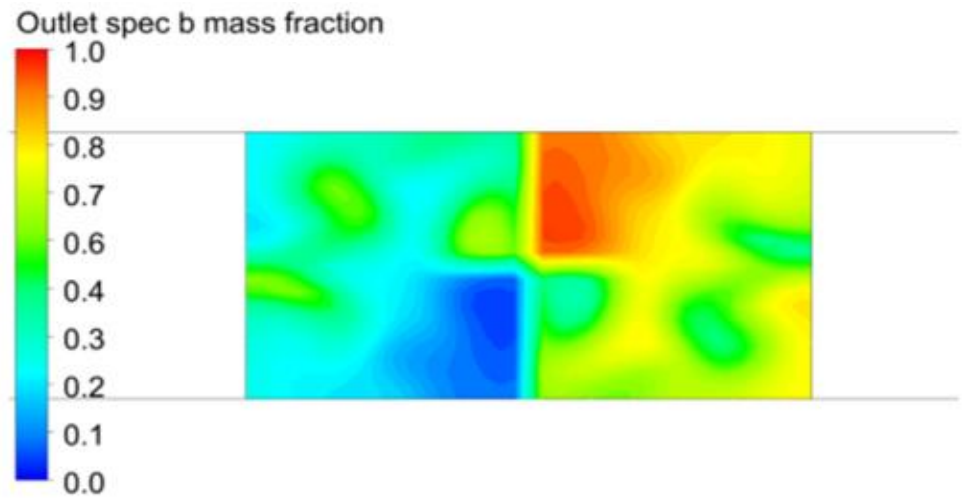

Figure 14. Outlet mass fraction contour of T-mixer with TDOs at $R e=345$

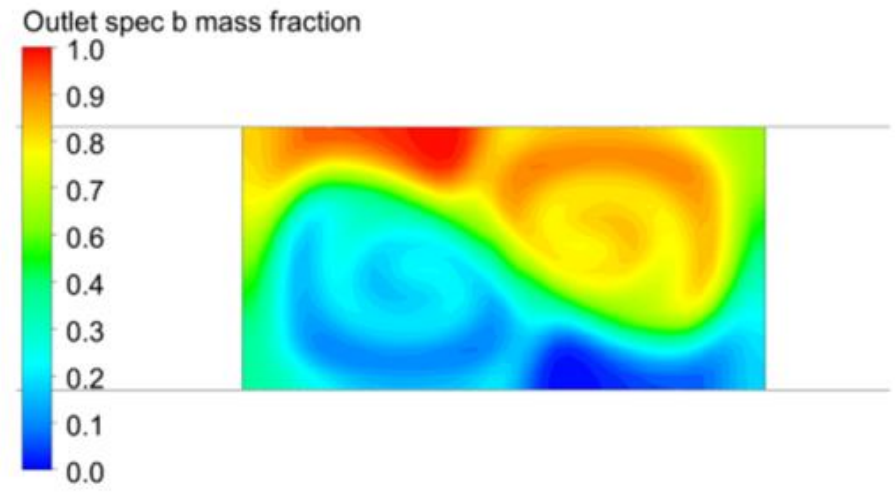

Figure 15. Outlet mass fraction contour of T-mixer at $R e=345$ 
International Journal of Mathematical, Engineering and Management Sciences

Vol. 5, No. 1, 147-160, 2020

https://doi.org/10.33889/IJMEMS.2020.5.1.013

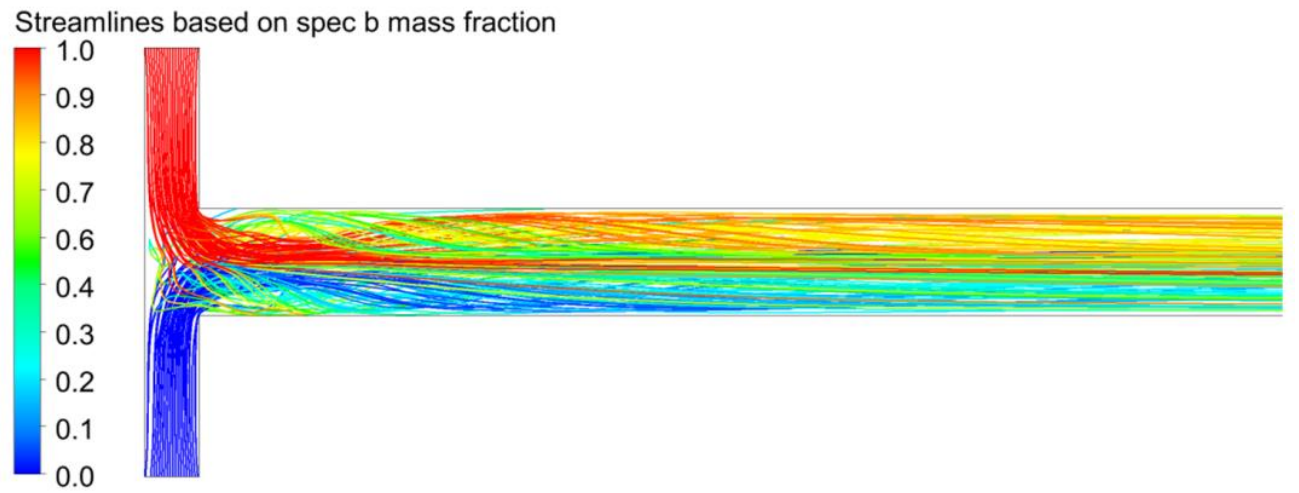

Figure 16. Streamlines from top view of T-mixer at $R e=345$

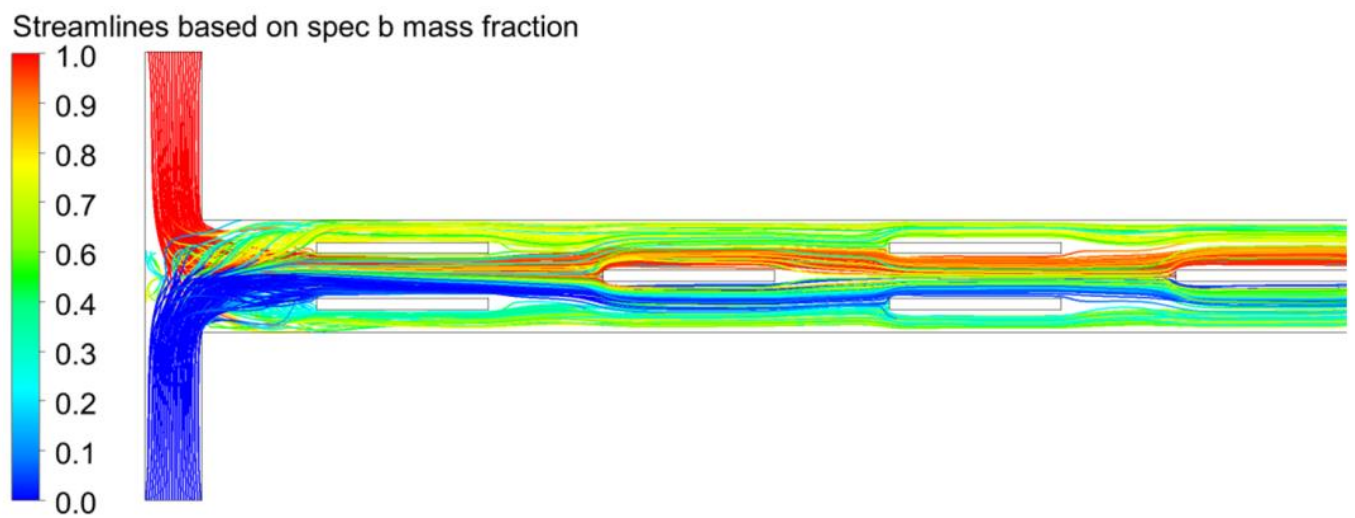

Figure 17. Streamlines from top view of T-mixer with TDOs at $R e=345$

\section{Conclusion}

The effect of Taylor dispersion type obstructions for the creation of velocity gradients in the flow of T-micromixer to improve its efficiency was investigated in the current study. It is found that at lower $\operatorname{Re}(0$ to 150$)$, the velocity gradients created in the flow due to the presence of TDO (Taylor Dispersion Obstruction) are smaller in amplitude and not significant enough to create the axial dispersion and enhance the mixing. In the $R e$ range of 100 to 150 , the vortex nature of the flow in T-mixer ends up damping the velocity gradients near TDOs and walls of the mixing channel. The increase in mixing performance with TDOs is negligible for the engulfment regime occurring in T-mixer for $R e$ of 160 to 220 . However, in higher $R e$ (250 to 350), due to the dominant inertial effects, better velocity gradients are created in the flow and a noteworthy increase in the performance of mixing is achieved with the presence of TDOs compared to conventional Tmixer. Therefore, it is concluded that the existence of Taylor Dispersion type obstructions in Tmixer exercises considerable influence and improves the mixing performance only at higher $R e$ flows $(R e>250)$. 
International Journal of Mathematical, Engineering and Management Sciences

Vol. 5, No. 1, 147-160, 2020

https://doi.org/10.33889/IJMEMS.2020.5.1.013

\section{Conflict of Interest}

The authors confirm that there is no conflict of interest to declare for this publication.

\section{Acknowledgement}

The authors would like to express their sincere thanks to the referee for their valuable suggestions and Dr. M. Raja

Vishwanathan for his support to improve the quality of research paper.

\section{References}

Bau, H.H., Zhong, J., \& Yi, M. (2001). A minute magneto hydro dynamic (MHD) mixer. Sensors and Actuators B: Chemical, 79(2-3), 207-215.

Bayer, T., Matlosz, M., \& Jenck, J. (2004). IMPULSE-Ein neuartiger Ansatz für die Prozessentwicklung. Chemie Ingenieur Technik, 76(5), 528-533.

Buchegger, W., Wagner, C., Lendl, B., Kraft, M., \& Vellekoop, M.J. (2011). A highly uniform lamination micromixer with wedge shaped inlet channels for time resolved infrared spectroscopy. Microfluidics and Nanofluidics, 10(4), 889-897.

Chan, C.K., Hu, Y., Takahashi, S., Rousseau, D.L., Eaton, W.A., \& Hofrichter, J. (1997). Submillisecond protein folding kinetics studied by ultrarapid mixing. Proceedings of the National Academy of Sciences, 94(5), 1779-1784.

Chen, C.K., \& Cho, C.C. (2008). Electrokinetically driven flow mixing utilizing chaotic electric fields. Microfluidics and Nanofluidics, 5(6), 785-793.

Chung, C.K., Shih, T.R., Wu, B.H., \& Chang, C.K. (2010). Design and mixing efficiency of rhombic micromixer with flat angles. Microsystem Technologies, 16(8-9), 1595-1600.

Dreher, S., Kockmann, N., \& Woias, P. (2009). Characterization of laminar transient flow regimes and mixing in T-shaped micromixers. Heat Transfer Engineering, 30(1-2), 91-100.

Engler, M., Kockmann, N., Kiefer, T., \& Woias, P. (2004). Numerical and experimental investigations on liquid mixing in static micromixers. Chemical Engineering Journal, 101(1-3), 315-322.

Freitas, S., Rudolf, B., Merkle, H.P., \& Gander, B. (2005). Flow-through ultrasonic emulsification combined with static micromixing for aseptic production of microspheres by solvent extraction. European Journal of Pharmaceutics and Biopharmaceutics, 61(3), 181-187.

Hardt, S., Drese, K., Hessel, V., \& Schönfeld, F. (2004, January). Passive micro mixers for applications in the micro reactor and $\mu$ TAS field. In ASME 2004 2nd International Conference on Microchannels and Minichannels (pp. 45-55). American Society of Mechanical Engineers. DOI: https://doi.org/10.1115/ICMM2004-2319.

Heshmatnezhad, F., Aghaei, H., \& Nazar, A.R.S. (2016). Parametric study of obstacle geometry effect on mixing performance in a convergent-divergent micromixer with sinusoidal walls. Chemical Product and Process Modeling, 12(1), 20160025. DOI: https://doi.org/10.1515/cppm-2016-0025.

Hessel, V., Hardt, S., Löwe, H., \& Schönfeld, F. (2003). Laminar mixing in different interdigital micromixers: I. Experimental characterization. AIChE Journal, 49(3), 566-577.

Hong, C.C., Choi, J.W., \& Ahn, C.H. (2004). A novel in-plane passive microfluidic mixer with modified Tesla structures. Lab on a Chip, 4(2), 109-113.

Lu, K.Y., Wo, A.M., Lo, Y.J., Chen, K.C., Lin, C.M., \& Yang, C.R. (2006). Three dimensional electrode array for cell lysis via electroporation. Biosensors and Bioelectronics, 22(4), 568-574. 
International Journal of Mathematical, Engineering and Management Sciences

Vol. 5, No. 1, 147-160, 2020

https://doi.org/10.33889/IJMEMS.2020.5.1.013

Nimafar, M., Viktorov, V., \& Martinelli, M. (2012). Experimental comparative mixing performance of passive micromixers with H-shaped sub-channels. Chemical Engineering Science, 76, 37-44.

Stroock, A.D., Dertinger, S.K., Ajdari, A., Mezić, I., Stone, H.A., \& Whitesides, G.M. (2002). Chaotic mixer for microchannels. Science, 295(5555), 647-651.

Suga, S., Nagaki, A., \& Yoshida, J.-I. (2003). Highly selective Friedel-Crafts monoalkylation using micromixing. Chemical Communications, (3), 354-355. DOI: 10.1039/B211433J.

Yaralioglu, G.G., Wygant, I.O., Marentis, T.C., \& Khuri-Yakub, B.T. (2004). Ultrasonic mixing in microfluidic channels using integrated transducers. Analytical Chemistry, 76(13), 3694-3698.

Zhang, C., Xing, D., \& Li, Y. (2007). Micropumps, microvalves, and micromixers within PCR microfluidic chips: Advances and trends. Biotechnology Advances, 25(5), 483-514. 